\title{
Introduction : Pour une approche pluridisciplinaire des usages politiques des cartes
}

\author{
Thomas Aguilera (Sciences Po Rennes - ARENES) \\ Francesca Artioli (Université Paris-Est-Créteil - Lab-Urba) \\ Lorenzo Barrault-Stella (CNRS - CRESPPA) \\ Emmanuelle Hellier (Université Rennes 2 - ESO) \\ Romain Pasquier (CNRS - ARENES - Chaire TMAP)
}

La carte est un instrument classique d'action publique : elle ne constitue pas seulement un outil de description de configurations spatiales, une technique de visualisation et d'objectivation d'un certain nombre de faits et de phénomènes, mais elle correspond surtout à une technologie de gouvernement porteuse de représentations, de conceptions des problèmes publics, de catégories, d'intérêts, d'hégémonie politique voire de hiérarchies sociales et produisant des effets politiques, sociaux et spatiaux. En cela, on peut dire qu'elle instaure un rapport spécifique entre gouvernants et gouvernés ${ }^{1}$. La carte de l'action publique renvoie également à l'organisation spatiale des services publics et des administrations (carte judiciaire, carte scolaire, carte militaire, etc.), dont elle donne une certaine représentation synchronique ou dynamique. Du fait de cette double acception d'instrument d'action publique et de représentation d'une organisation spatiale, la carte est un objet particulièrement intéressant pour penser les mutations de l'action publique sur les territoires, et plus particulièrement les reconfigurations des relations entre décideurs, metteurs en œuvre et gouvernés. Cet ouvrage ${ }^{2}$ se donne en ce sens pour objectif de remettre les cartes de l'action publique sur le métier, dans une perspective interdisciplinaire, en s'intéressant aux transformations des rapports entre politiques publiques et usagers, entre État et collectivités locales, entre gouvernants et gouvernés, dont elles sont à la fois porteuses et témoins sur des territoires donnés. Les cartes peuvent être envisagées comme des politiques constitutives en ce qu'elles participent aux processus de réforme internes aux États et portant sur ses mêmes structures. En ce sens, on peut étudier les transformations des cartes de l'action publique - au sens des mutations spatiales et organisationnelles des services publics et des administrations -, ainsi

\footnotetext{
1 Pierre Lascoumes (dir.), « Gouverner par les cartes », Genèses, vol. 3, n 68, 2007.

2 Cet ouvrage est partiellement issu d'un colloque organisé les 5 et 6 avril 2018 à Paris (CNRS, Site Pouchet) qui faisait suite à la rencontre entre des programmes de recherche menés au sein de différentes institutions depuis quelques années : d'un côté à Paris avec le Lab'Urba (Francesca Artioli) et le CRESPPA (Lorenzo BarraultStella), et, de l'autre à Rennes avec la Chaire Territoires et mutations de l'action publique de Sciences Po Rennes et le laboratoire ESO (séminaire «Gouverner par les cartes », Thomas Aguilera, Emmanuelle Hellier, Romain Pasquier, Marc Rouzeau).
} 
que les effets de ces réformes sur la mise en œuvre de l'action publique et, plus largement, sur les groupes sociaux qui y sont confrontés. Il s'agira d'interroger les logiques institutionnelles, bureaucratiques et politiques de la réorganisation spatiale de l'action publique, les modalités de réforme, les modes de légitimation, les mobilisations et les conflits qu'elles peuvent (ou non) susciter. La transformation des cartes de l'action publique suppose de comprendre pourquoi, selon quelles expertises, quels choix politiques et quelles données, quelles pratiques et représentations des gouvernés, les découpages, les périmètres d'action et les implantations des institutions publiques sont redessinés. Cette perspective permettra d'étudier ce que les mutations des cartes de l'action publique font à l'État, aux collectivités territoriales, aux groupes professionnels et aux groupes sociaux qui se mobilisent localement pour défendre des intérêts, pour revendiquer une forme d'équité territoriale ou encore, pour défendre une identité et une histoire. En même temps, les cartes ne sont pas qu'un vecteur de changement, mais, en tant que dispositif matériel de gouvernement, peuvent en retour participer à consolider l'action publique et à l'inscrire dans le temps. En effet, à la fois la production d'un savoir cartographique et l'intervention publique sur les territoires ont un pouvoir instituant, en ce qu'elles participent à l'institutionnalisation de l'action publique et de l'autorité politique (soit-elle l'État, une collectivité territoriale ou l'Union européenne) qui est en est à l'origine. La carte est potentiellement performative.

Dans cette introduction, nous proposons une brève synthèse des travaux réalisés en science politique, en sociologie et en géographie sur les cartes afin de justifier notre approche pluridisciplinaire et d'étayer notre problématisation singulière. Celle-ci est articulée autour de trois grands axes: les cartes participent à l'institutionnalisation de centres politiques et de territoires; elles cristallisent des conflits et participent de la fabrique de représentations des espaces et des problèmes publics; elles représentent des vecteurs de changement de l'action publique tout autant qu'elles en constituent des miroirs, s'inscrivant par la même dans certaines reconfigurations des structures étatiques comme des modes de gouvernement. 


\section{Une approche résolument pluridisciplinaire}

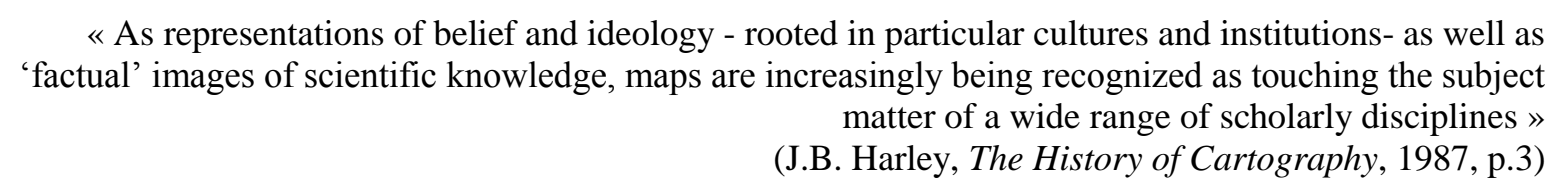

Le présent ouvrage est résolument pluridisciplinaire : il réunit des contributions de politistes, de sociologues, de géographes qui, chacun-e à leur manière (selon des cadrages théoriques et des méthodes variées), proposent une analyse de la fabrique et de l'usage politiques des cartes. À ce titre, l'ouvrage s'adresse à un public pluriel et élargi. L'objectif d'interdisciplinarité dépasse la juxtaposition d'approches disciplinaires, les croiser afin d'en proposer une combinaison inédite voire de modifier les disciplines en question - est plus difficile à atteindre. Mais le colloque dont est issu le présent ouvrage collectif fut un premier pas vers un dialogue autour d'un même objet et à la prise en compte par chacun des auteur-e-s de diverses perspectives théoriques et méthodologiques. La présente introduction justifie et confirme cette perspective pluridisciplinaire et invite à une lecture générale interdisciplinaire de l'ouvrage : parcourant les chapitres avec un regard transversal, le lecteur pourra reconstituer une analyse unifiée sur la base d'une mosaïque pluridisciplinaire. Ainsi, nous proposons tout d'abord de revenir sur les perspectives proposées par chacune des trois grandes disciplines évoquées (géographie, science politique, sociologie) afin d'y collecter les points saillants et les apports qui pourraient s'étayer et se consolider mutuellement. La carte et la cartographie ont été appréhendées et étudiées de diverses manières, mais un point commun émerge rapidement : les sciences sociales sont légitimes à s'en saisir car la cartographie n'est pas une opération automatisée de représentation mais relève bien des techniques du pouvoir politique, des contextes et des finalités de son usage, et à ce titre de l'action publique.

C'est donc une interrogation sur l'usage politique des cartes, dans l'action publique et dans l'action collective, qui constitue le fil conducteur et la problématique générale de cet ouvrage. Quelle que soit l'échelle de gouvernement, la connaissance des territoires, des populations et des enjeux sociaux est un enjeu pour les autorités publiques ${ }^{3}$. Le point de vue de l'État sur les groupes qu'il prétend gouverner s'exprime et se réfracte à travers les représentations cartographiques que l'état des savoirs permet de produire ${ }^{4}$. Symétriquement, le tracé des frontières cartographiques en

3 John Pickles, A history of spaces. Cartographic reason, mapping and the geo-coded world, Londres, Routledge, 2004. Au-delà du cas des seules cartes : Michel Foucault, La volonté de savoir, Paris, Gallimard, 1976.

4 James C. Scott, Seeing Like a State: How Certain Schemes to Improve the Human Condition Have Failed, Yale University Press, 1999. 
matière de définition des territoires administrés est central dans la construction des identités collectives $^{5}$, mais ses décalages avec les territoires vécus et les territoires fonctionnels nécessitent une certaine souplesse dans la mise en œuvre des politiques publiques ${ }^{6}$. Les usages politiques et sociaux des cartes relèvent ainsi d'enjeux majeurs à la fois pour les institutions publiques et pour les gouvernés. Leurs appropriations, multiples et potentiellement contradictoires, s'inscrivent dans un espace des possibles particulièrement élargi.

\subsection{La carte, reflet et instrument du pouvoir sur les territoires}

Faire l'histoire de la cartographie est une entrée privilégiée pour penser l'histoire sociale et politique des civilisations humaines ainsi que leurs rapports aux territoires ${ }^{7}$. Si historiens et anthropologues ont montré que la carte peut être considérée comme un support universel de communication - ayant parfois même précédé (voire supplanté) l'écriture - l'histoire de la cartographie telle que développée par le grand géographe et historien de la cartographie Brian Harley et ses collègues a permis d'historiciser un support matériel, d'en présenter une histoire non linéaire et non téléologique et dépassant l'histoire d'une technique. Certes la carte a bénéficié des avancées scientifiques et technologiques (astronomie, géographie...), mais il s'agit surtout d'en questionner les usages et la portée pour les sociétés au-delà de sa dimension utilitaire et scientifique, en ne la réduisant pas à ses considérations techniques ${ }^{8}$ : en tant qu'outil de visualisation et de figuration d'un espace, la carte n'est ni neutre, ni déconnectée des contextes sociaux et politiques dans lesquels elle est conçue, développée et utilisée. Si la connaissance fine des techniques de production de la carte est une condition nécessaire à sa compréhension plus globale, le travail des sciences sociales est davantage de rendre compte des relations sociales, des enjeux politiques voire culturels qu'elle renferme et cristallise.

Tout d'abord, la carte constitue une forme de langage, porteuse d'histoires et de représentations. Cartographier en vue de donner l'image la plus exacte de la surface terrestre est déjà un exercice de déformation/transformation par rapport à l'objet d'origine, dans la mesure où il faut utiliser une méthode de projection (d'une surface ronde vers une surface plane) et appliquer un coefficient de réduction (l'échelle). Plusieurs méthodes de projection existent, plusieurs échelles de représentation sont possibles. En outre, les conditions de réalisation, compétences et

5 Claude Raffestin, Pour une géographie du pouvoir, Lyon, ENS éditions, 1980.

6 Annie Bleton-Ruget, Nicole Commerçon, Philippe Gonod (dir.), Territoires institutionnels, territoires fonctionnels, Institut Val-de-Saône-Mâconnais, 2006.

7 John Brian Harley \& David Woodward (eds.), The History of Cartography. Cartography in Prehistoric, Ancient and Medieval Europe and the Mediterranean, Chicago, The University of Chicago Press, 1987.

8 Peter Gould et Antoine Bailly, Le pouvoir des cartes. Brian Harley et la cartographie, Paris, Academica, 1995. 
outils techniques à disposition varient selon les contextes, si bien que pour le même usage final (une carte pour la randonnée pédestre par exemple), on obtient un résultat différent selon qu'on soit cartographe professionnel à l'IGN (la carte standard IGN au 1/25000e) ou dessinateur amateur (croquis adapté au public visé). Néanmoins, la carte revêt une grande valeur heuristique. En effet, produire une carte révèle chez celui ou celle qui la conçoit, la réalise et la diffuse, des schèmes de sa vision d'un objet ; plus largement, elle peut dire quelque chose de sa représentation du monde. Le fait de placer l'Europe au centre sur un planisphère est symptomatique des cartes produites dans les pays européens (même chose pour les pays des autres continents, qui placent leur continent d'appartenance au centre). On peut détecter la marque de l'auteur dans les cartes créatives, de contrées imaginaires ou de lieux utopiques présentes dans la littérature ${ }^{9}$, mais aussi dans les cartes mentales des espaces de vie produites par des habitants ${ }^{10}$, des enfants ${ }^{11}$, des usagers lors d'enquêtes en sociologie ou en psychologie environnementale ${ }^{12}$. La cartographie est en ce sens une traduction graphique de schémas cognitifs et perceptifs qui structurent et différencient le rapport des individus à l'espace en fonction de leur(s) statut(s) et appartenances sociales ${ }^{13}$.

À ce titre, la carte doit être réinsérée dans un double contexte social et politique : « la carte est elle-même une biographie en tant qu'objet matériel conçu, fabriqué et utilisé à une certaine époque ${ }^{14}$. La carte "parle » tant pour le concepteur que pour l'usager, renvoyant à ce que les géographes et aujourd'hui certains acteurs des politiques publiques nomment les «cartes mentales ${ }^{15}$ : la représentation des espaces vécus n'est pas neutre et permet de rendre compte de perceptions, d'usages et de cadres cognitifs. De même, la carte évoque des paysages qui peuvent varier selon les individus, les groupes, les sociétés en intégrant même parfois la dimension culturelle. Enfin, la carte raconte l'histoire collective de sociétés ou de groupes spécifiques, et évoque ainsi des événements et des personnages : elle fait exister le passé comme constitutif de

9 Italo Calvino, Les villes invisibles, Paris, Seuil, 2013.

10 Kevin Kynch, The Image of the City, Harvard-Londn, MIT Press, 1960.

11 Les travaux de Jean Piaget et ses collègues furent fondateurs en ce domaine bien que critiqués et amendés par la suite (Jean Piaget et Barbel Inhelder, La représentation de l'espace chez l'enfant, Paris, PUF, 1948). Plus récemment, les cartes mentales ont été mobilisés comme outils de représentation et d'enquête sur le rapport des enfants à l'espace: Thierry Ramadier et Sandrine Depeau, «Approche méthodologique (JRS) et développementale de la représentation de l'espace urbain quotidien de l'enfant », in Isabelle Danic, Olivier David, Sandrine Depeau (dir.), Les enfants et les jeunes dans les espaces quotidiens, Rennes, Presses Universitaires de Rennes, p. 61-74.

12 Thierry Ramadier et Anne-Christine Bronner, « Knowledge of the environment and spatial cognition: JRS as a technique for improving comparisons between social groups », in Environment and Planning B: Planning and Design, 2006, 33(2), pp. 285-299.

13 Pierre Dias, Thierry Ramadier, « Representational Structures as Stances: Examining Relationships to the City Under the Lens of Socio-Spatial Representations », in International Review of Social Psychology, 2018, 31(1) : 24, p. 1-13.

14 Brian Harley, «La carte en tant que biographie : réflexions sur la carte du service cartographique de l’État. Newton Abbot, Devonshire CIX, SE », in Peter Gould et Antoine Bailly, Le pouvoir des cartes. Brian Harley et la cartographie, op.cit., p. 13.

15 Peter Gould, \& R. White, Mental maps, Harmondsworth, Penguin, 1974. 
notre présent ${ }^{16}$. Dans le chapitre 8 de cet ouvrage, Jeanne Toutous montre ainsi comment le mouvement régionaliste breton est porteur tout à fois d'une histoire et d'une carte régionale alternative à celle de l'Etat central, celle de la Bretagne à cinq départements.

La géographie de la fin du XXème siècle et l'histoire politique ont depuis longtemps exploré et documenté le lien étroit entre espace et pouvoir, et la carte y joue un rôle particulier. On peut dessiner un continuum d'appréhension entre deux pôles : la carte comme reflet ou comme instrument politique. D'un côté, la carte constitue un reflet du contexte politique de conception et de réception. À partir du moment où l'on considère que l'espace reflète des rapports sociaux et politiques ${ }^{17}$, on peut saisir la carte comme un miroir permettant au chercheur, et notamment à l'historien, d'identifier des traces spatialisées des rapports de force, des hiérarchies, des conflits entre groupes sociaux, des tensions entre des intérêts ${ }^{18}$. D'un autre côté, on peut considérer la carte comme un instrument au service des gouvernants pour imposer des choix politiques et pour réguler les sociétés. La carte a servi à faire la guerre, à coloniser des territoires inconnus ${ }^{19}$, à consolider des empires $^{20}$ et à stabiliser des frontières ${ }^{21}$. L'État ayant longtemps été considéré comme l'un des seuls commanditaires de cartes, cette dimension command and control, stratégiste, instrumentale et top-down de la carte s'est bien souvent imposée, d'autant plus que la dimension symbolique est également forte, faisant de l'identification des «distorsions délibérées du contenu des cartes » et des «amplifications sélectives » ${ }^{22}$ (manipulation d'échelles, choix de couleurs suggestives, omissions) l'une des principales missions du chercheur en sciences sociales.

Au-delà des enjeux de représentation du pouvoir de l'Etat, l'activité cartographique a permis, avec la statistique ${ }^{23}$, de contrôler les populations pour réguler leur implantation, leurs activités, leurs échanges marchands, pour collecter des impôts et bien sûr pour répartir et sécuriser la propriété foncière. Les historiens font effectivement de la mise en place de cadastres un point

16 Denis Wood, The power of maps, Guilford Press, 1992, p. 1.

17 Sylvie Tissot et Franck Poupeau, «La spatialisation des problèmes sociaux », Actes de la recherche en sciences sociales, vol. 4, n¹59, 2005, p. 4-9 ; Edward Soja, «La ville et la justice spatiale », Justice spatiale | spatial justice, $\mathrm{n}^{\circ} 1,2009$.

18 Brian Harley, «Maps, knowledge and Power », in Denis Cosgrove \& Stephen Daniels, The Iconography of landscape: Essays on the Symbolic representation, design and use of past environments, New York, Cambridge University Press, 1988.

19 James Scott, Seing Like a State, op cit.; Hélène Blais, Mirages de la carte. L'invention de l'Algérie coloniale, Paris, Fayard, 2014.

20 Alexander J. Kent, Soatkin Vervust, Imre J. Demhardt, Nick Millea, (dir.), Mapping Empires: Colonial Cartographies of Land and Sea, Springer, 2020.

21 David Buisseret, Ingénieurs et fortifications avant Vauban. L'organisation d'un service royal aux XVIèmeXVIIème siècles, Paris, Edition du Comité des travaux historiques et scientifiques, 2002.

22 Brian Harley, «Cartes, savoir et pouvoir », in Peter Gould et Antoine Bailly, Le pouvoir des cartes, op.cit., p.33.

23 Alain Desrosières, « Historiciser l'action publique : l'Etat, le marché et les statistiques », in Pascale Laborier \& Dany Tron (dir.), Historicités de l'action publique, Paris, PUF, 2003, p. 207-221. 
nodal de la construction des Etats en lien avec les grandes transformations économiques (révolutions agraires et industrielles) et des capitalismes, notamment en Europe ${ }^{24}$. Sur une perspective de temps long, on peut considérer que la cartographie cadastrale a participé au passage du monde féodal à la révolution industrielle puis au monde capitaliste moderne ${ }^{25}$. Pour certains, la carte cadastrale fut même l'un des instruments au cœur - et au service - de l'affirmation des Etats européens et des Empires $^{26}$. De même, les historiens et géographes marxistes considèrent le mouvement des «enclosures » - mises en place en Angleterre dès le XIIème siècle mais surtout entre le XVIème et le XVIIIème siècles - comme l'une des sources de la révolution agraire (fin d'une agriculture traditionnelle par dépossession les petits agriculteurs) puis de la révolution industrielle (constitution d'une main d'œuvre importante au service des industries des villes) : si initialement les enclosures viennent pallier le manque de cadastre, ce processus d'《accumulation par dépossession ${ }^{27}$ va progressivement s'appuyer sur les relevés topographiques et les représentations cartographiques des divisions parcellaires pour stabiliser les délimitations territoriales des propriétés foncières privées et donc sécuriser l'exploitation, l'échange et l'accumulation des ressources ${ }^{28}$. La carte cadastrale a été utilisée à partir du XVIIIème siècle par les Etats européens à la fois comme instrument de relevé des parcelles et transformation des divisions foncières ${ }^{29}$.

24 Marie-Vic Ozouf-Marignier, «La division du territoire : limites naturelles et limites politiques », in Andrée Corvol (éd.), La nature en révolution (1750-1800), Paris, 1992, p.126-133 ; Florence Bourillon, Nadine Vivier (dir.), La mesure cadastrale. Estimer la valeur du foncier, Rennes, PUR, 2012.

25 Les archéologues ont retrouvé des formes de cadastres il y a 3000 ans av. JC en Mésopotamie, mais aussi dans l'Antiquité Egyptienne et Romaine, tout en mettant en évidence la diversité de formes, d'instrumentation et de significations qu'elles pouvaient prendre. Au Moyen-Âge en Europe, des registres, parfois accompagnés de plans, ont été mis en place mais de façon fragmentaire et dispersée, et les institutions religieuses ont joué un rôle important dans la construction de subdivisions territoriales. Les administrations des Empires coloniaux ont ensuite développé des techniques de relevés topographiques pour explorer et conquérir, mais aussi pour organiser et exploiter les territoires, donnant lieu là aussi à des embryons de cartes cadastrales et de statistiques d'Etat qui, en retour, ont influencé la construction des Etats modernes européens. L'apport de Napoléon Bonaparte en France au début du XIXème siècle (1807) repose sur la systématisation et rationalisation de l'association entre registres et cartographie pour couvrir l'ensemble d'un territoire et servir de base à l'ancrage territorial de l'administration et de la politique fiscales. Puis, partout dans l'Europe du XIXème siècle, bénéficiant d'avancées techniques, scientifiques mais aussi administratives, l'activité cartographique s'est rationalisée, bureaucratisée et professionnalisée, au service d'Etats qui utilisent les cartes comme des outils de gouvernement majeurs pour collecter des taxes, sécuriser la propriété privée et intensifier l'exploitation des ressources naturelles.

26 Pour une histoire longue de la cartographie cadastrale et de son rôle dans la construction des Etats, se référer à la somme de Roger J. P Kain \& Elizabeth Baigent, The Cadastral Map In The Service Of The State: A History Of Property Mapping, Chicago, University of Chicago Press, 1992.

27 David Harvey, «The Future of the Commons », in Radical History Review, n¹09, 2011, p. 101-107.

28 Ellen Meiksin Wood, «The Agrarian Origins of Capitalism », Monthly Review, July 1998, p. 14-31.

29 L'Etat a joué un rôle plus ou moins direct dans la mise en œuvre : par exemple, en Angleterre, si l'usage des cartes est requis par le Parlement à partir du XVIIIème siècle, les cartes sont d'abord réalisées par des enquêteurs privés alors qu'en Suède et en Finlande, les agents de l'Etat réalisaient ces relevés mais financés par les propriétaires (Roger J. P Kain \& Elizabeth Baigent, The Cadastral Map In The Service Of The State, op. cit., p. $334)$. 
Ainsi, dans un continuum entre ces deux pôles (reflet des rapports socio-politiques / outil au service des gouvernants), les travaux s'accordent surtout sur le fait que la carte a toujours constitué une ressource de légitimation politique qui rend performative la conquête de territoires et l'intégration territoriale des différentes formes d'autorité politique au premier rang desquelles de l'État ${ }^{30}$. Qu'elle soit source, vectrice ou conséquence de décisions politiques, la carte contribue ainsi à «discipliner» l'espace en lien avec les transformations des économies capitalistes et des reconfigurations politiques. Enfin, la carte représente une forme de savoir qui, dans une perspective foucaldienne largement appropriée par la géographie ${ }^{31}$, est porteur de pouvoir : la conception cartographique nécessite et affirme la détention de l'information et de la donnée sur les territoires et les populations. La carte n'est donc jamais neutre et appelle à ce titre à être saisie par une approche de sciences sociales attentive à son appropriation par les acteurs et à ses effets socio-politiques, sans s'en tenir à ce qu'elle donne à voir : décrypter et déconstruire les processus de «naturalisation » de la carte comme technique de visualisation est nécessaire pour en révéler son instrumentation au service du pouvoir. Les travaux développés en science politique et notamment par les sociologues de l'action publique ont développé des outils conceptuels pour formaliser ces réflexions en considérant la carte comme un instrument d'action publique à part entière mais aussi comme un instrument parmi d'autres, évitant ainsi d'en réifier la portée dans la construction sociopolitique des territoires.

\subsection{La carte, un instrument d'action publique comme les autres}

Les politistes se sont saisis plus tardivement de la carte comme objet à part entière mais elle fut d'emblée saisie dans la lignée de l'analyse des instruments d'action publique ${ }^{32}$, c'est-à-dire en mettant à distance la visée rationaliste et gestionnaire : la carte correspond à un dispositif matériel (technique et social) qui participe à la régulation de la relation gouvernantsgouvernés, sur de territoires donnés, en fonction des représentations dont il est porteur ${ }^{33}$. Considérer la cartographie comme faisant partie d'un processus d'instrumentation de l'action publique $^{34}$ incite à porter la focale sur l'opérationnalisation de l'action publique, sur la

30 Saskia Sassen, Territory, Authority, Rights: From Medieval to Global Assemblages, Princeton University Press, 2006 ; Stuart Elden, The Birth of Territory, Chicago, University of Chicago Press, 2013.

31 «Questions à Michel Foucault sur la géographie », Hérodote, n 1, 1976, p. 71-85.

32 Pierre Lascoumes, « Gouverner par les cartes », op. cit.

33 Pierre Lascoumes et Patrick Le Galès, «L'action publique saisie par ses instruments », Gouverner par les instruments, Paris, Presses de Sciences Po, 2004, p. 13.

34 On peut définir l'instrumentation de l'action publique comme «l'ensemble des problèmes posés par le choix et l'usage des outils (des techniques, des moyens d'opérer, des dispositifs) qui permettent de matérialiser et 
matérialité de la mise en œuvre des programmes d'action gouvernementaux, au-delà des objectifs affichés et des discours volontaristes qui mettent en scène l'innovation afin de tracer les changements ${ }^{35}$ et de distinguer ce qui relève de l'innovation ou du recyclage institutionnel $^{36}$. Le chapitre de Nicolas Larchet (chapitre 7 de cet ouvrage) s'inscrit dans cette démarche de repérage du changement et plus précisément de recadrages des problèmes publics par l'analyse de l'instrumentation. L'approche par les instruments permet ainsi de tracer les effets concrets des politiques publiques sur les groupes sociaux comme sur les territoires.

La carte n'est ni la photographie d'un espace, ni un ensemble ordonné d'aires, de points et de lignes ; elle est avant tout représentation de données, à travers un condensé de contraintes et de choix techniques réalisés par des acteurs ; ces derniers ont dû s'accorder tantôt dans le conflit, tantôt dans le compromis - sur la façon de représenter des problèmes spatiaux et sociaux ${ }^{37}$. De ressort politique, ces choix techniques ont aussi des implications politiques au sens où ils produisent de fait de la distinction entre bénéficiaires et victimes (qu'ils soient individus, groupes sociaux, administrations, entreprises privées ou territoires) par l'élaboration de seuils ou de zones, mais aussi de catégories qui diffusent et participent à la conception de représentations sociales et politiques. C'est ce qui est montré par Christine Zanin dans son chapitre sur les cartes des politiques européennes (chapitre 3). Des choix apparemment anodins et dépolitisés peuvent pourtant avoir des conséquences majeures sur la conception et la mise en œuvre des politiques publiques comme le montrent dans cet ouvrage Magali Nonjon, Julien O’Miel et Julien Talpin à propos de la géographie prioritaire (chapitre 5), dans le même sens que les conclusions de Philippe Estèbe quant aux quartiers de la politique de la ville dans les années $2000^{38}$.

Enfin, en fonction de l'échelle d'analyse et du type de carte, la carte pourrait être considérée comme un «méta-instrument ${ }^{39}$ (Hood, 1983) en tant que dispositif qui coordonne une diversité d'autres instruments, d'acteurs (concepteurs, utilisateurs,

d'opérationnaliser l'action gouvernementale »: Charlotte Halpern, Pierre Lascoumes et Patrick Le Galès, L'instrumentation de l'action publique, Paris, Presses de Sciences Po, 2014, p. 17.

35 Bruno Palier, «Les instruments, traceurs du changement. Les politiques des retraites en France », in Pierre Lascoumes et Patrick Le Galès (dir.), Gouverner par les instruments, op. cit, p. 273-300.

36 Thomas Aguilera, «Innover par les instruments ? Le cas du gouvernement des squats à Paris », in Charlotte Halpern, Pierre Lascoumes et Patrick Le Galès, L'instrumentation de l'action publique, op.cit, p. 417-443.

37 Jean-Pierre Le Bourhis, « Du savoir cartographique au pouvoir bureaucratique. Les cartes des zones inondables dans la politique des risques (1970-2000) », in Genèses, vol. 3, n68, p. 75-96.

38 Philippe Estèbe, «Les quartiers, une affaire d'État. Un instrument territorial », in Pierre Lascoumes et Patrick Le Galès (dir.), Gouverner par les instruments, Paris, Presses de Sciences Po, 2004, p. 47-70.

39 Christopher Hood, The Tools of Government, London, Mac Millan, 1983. 
observateurs), d'outils et de techniques ${ }^{40}$. Cette proposition qui consiste à déconstruire l'unité de la carte a deux conséquences. D'une part, elle incite systématiquement à ne pas réifier la carte et ne pas considérer qu'elle produit par elle-même des effets : elle ne représente qu'un instrument d'action publique parmi d'autres et en lien avec d'autres, au premier rang desquels la statistique ${ }^{41}$. Comme Antoine Courmont (chapitre 1) et Boris Merisckay (chapitre 4) le montrent dans leurs chapitres respectifs dans cet ouvrage, la construction des cartes nécessite la production et la mise à disposition de base de données statistiques géolocalisées qui doivent être ensuite territorialisées, entrainant d'ailleurs des coûts importants de conversion, de circulation et de coordination. D'autre part, elle incite à voir la carte comme résultant d'un bricolage et non pas d'un processus linéaire de représentation d'un problème en vue de sa résolution. Les acteurs piochent, parfois de façon chaotique, des outils existants dans des boites à outils, négocient, ajustent, recyclent et justifient a posteriori leur choix comme une sélection raisonnée en mobilisant la légitimité scientifique, notamment issue de la statistique publique et de l'économie. Les politiques d'aménagement du territoire en France, singulièrement celles visant le rééquilibrage des emplois et des activités de la région parisienne vers le reste du pays, s'appuient sur la diffusion des savoirs de l'économie géographique, utilisés de façon sélective par les élites politico-administratives de l'Etat souvent en concurrence entre-elles ${ }^{42}$. De plus, les acteurs participant à la conception des cartes peuvent être multiples, là aussi à l'encontre d'une conception top-down dont les clés sont détenues par l'État et les élites technocratiques. L'approche par les instruments d'action publique combinée à une sociologie politique des publics et des formes de participation citoyenne ont alors permis de montrer que la carte pouvait non seulement être appropriée, contestée voire conçue par les gouvernés.

La carte est donc un instrument puissant au service de la décision, de sa légitimation et de sa communication : par le symbolisme et la sémiologie graphique, elle donne à voir et rend visible des effets performatifs importants. Pourtant, elle n'est qu'un instrument parmi d'autres et ne doit pas être isolée dans une analyse des transformations de l'action publique sur les territoires au risque d'en réifier et d'en exagérer les effets comme les innovations. Grâce à une approche pluridisciplinaire, cet ouvrage propose de faire le lien entre la carte de l'action

\footnotetext{
40 Pour préciser la distinction entre instruments, outils et techniques se référer à l'introduction de Gouverner par les instruments. Ici, on pourrait distinguer la cartographie (procédure d'instrumentation), la carte (instrument), l'outil (échelle, légende), la technique (zonage).

41 Alain Desrosières et Emmanuel Didier, Prouver et gouverner, Paris, La Découverte, 2014.

42 Pauline Prat, L'institutionnalisation de l'action de l'État en région parisienne. Du plan Prost à la police d'agglomération: quand l'État administre, aménage et surveille la région-capitale, Thèse de science politique. Paris : Institut d'Etudes Politiques de Paris, 2012.
} 
publique comme vecteur d'intégration territoriale, d'institutionnalisation et de réformes des organisations politiques sur les territoires et de conflits entre gouvernants et gouvernés.

\section{Cartes et fabrique des espaces politiques}

L'instrumentation cartographique joue un rôle majeur dans la fabrication multiscalaire des espaces politiques. En construisant une représentation d'un espace et en matérialisant ses frontières, la carte contribue à son institutionnalisation en le faisant exister sur la base de critères économiques, sociaux, culturels ou politiques. Comme l'ont montré tant les travaux de sociologie historique de l'État ${ }^{43}$ que les travaux sur l'institutionnalisation de 1'Union Européenne ${ }^{44}$, la structuration d'une autorité politique et d'un espace politique y afférent se produit par la multiplication et l'intensification des relations politiques, économiques et sociales à cette échelle. Cela participe de l'institutionnalisation d'un centre politique et d'un territoire, et organise les comportements des acteurs (individuels et collectifs) en son sein. Cette fabrication d'un espace politique (en premier lieu, l'État nation) est un processus de longue durée auquel participe une multiplicité de phénomènes économiques, politiques et sociaux ${ }^{45}$. Comme il sera montré par plusieurs chapitres de cet ouvrage, la carte non seulement donne à voir un espace, mais contribue aussi à l'institutionnaliser. La cartographie, sa production et sa publicisation, permet aux constructeurs d'ordres politiques de « naturaliser » un territoire, de matérialiser ses frontières et de finalement donner à voir l'exercice du monopole de la violence physique légitime. La production de cartes peut alors être lue comme une des composantes du « pouvoir infrastructurel ${ }^{46}$ de l'État, défini comme la capacité de celui-ci à pénétrer la société et à mettre en œuvre des décisions politiques qui l'organisent, à travers une bureaucratie, des élites, des infrastructures, des taxes, mais aussi la production et la mobilisation massive d'informations sur les individus et les espaces, y compris par la cartographie et la statistique. Ces procédures d'instrumentation sont utilisées pour établir (ou remettre en cause) des découpages territoriaux, pour allouer des ressources ou

43 Bertrand Badie et Pierre Birnbaum, Sociologie de l'État, Paris, Hachette, 1982 ; Charles Tilly, Coercion, Capital, and European States, Ad 990-1992, New edition., Blackwell Publishers, 1993.

44 Alex Stone Sweet, Wayne Sandholtz, Neil Fligstein, eds, The institutionnalization of Europe, Oxford, Oxford University Press, 2001 ; Sven Steinmo, Kathleen Thelen, Franck Longstreth, dir., Structuring Politics : Historical Institutionalism in Comparative Perspective, Cambridge, Cambridge University Press, 1992.

45 Caitríona Carter et Romain Pasquier, «Introduction: Studying Regions as 'Spaces for Politics': Re-thinking Territory and Strategic Action », Regional \& Federal Studies, 2010, vol. 20, n 3, p. 281-294.

46 Michael Mann, «The autonomous power of the state: its origins, mechanisms and results », European Journal of Sociology / Archives Européennes de Sociologie, 1984, vol. 25, nº 2, p. 185-213. 
cibler des publics de politiques sectorielles, mais sont également mobilisées dans la construction d'appartenances et d'identités collectives.

Or, les espaces politiques font l'objet de luttes sociopolitiques, et leur institutionnalisation n'est jamais stable ni définitive. La construction de l'État nation est à cet égard particulièrement illustrative $^{47}$, car la construction étatique d'un centre génère des résistances et/ou des contremobilisations des périphéries. Les cartes participent à ces luttes en produisant des récits scalaires alternatifs. Par exemple, en France, la Révolution de 1789 met fin à la mosaïque territoriale héritée de l'Ancien régime, avec la création de la nouvelle carte départementale, devant garantir la participation et l'égalité de chacune des parties au sein du « grand tout $»^{48}$. Les régimes politiques qui se succèdent au XIXe et XXe siècles utilisent à loisir cet espace départemental comme un relais efficace pour le contrôle politique et la gestion publique. Et pourtant, dès la seconde moitié $\mathrm{du}$ XIXe siècle, les premières contestations du centralisme donnent naissance à représentations spatiales et des récits scalaires alternatifs. Paradoxalement, cette lutte politique contre la départementalisation reçoit un soutien inattendu venant de la sphère étatique. La mise en statistique de la France et la cartographie de plus en plus précise révèle, en effet, un tableau contrasté du pays, heurtant directement la conception unitaire héritée de la Révolution. Une des conséquences est la remise en cause du département jugé trop restreint en considération des unités économiques et culturelles. Selon la pensée positiviste qui se développe à l'époque, la division territoriale doit se faire à la lumière de faits scientifiques : Auguste Comte, parmi les premiers, proposera alors une répartition de la France en 17 régions ${ }^{49}$. Plus tard, le père fondateur de la géographie française, Paul Vidal de la Blache, construira également une image spatiale de la nation marginalisant le département au profit d'un maillage territorial alternatif, en régions et en pays décrits comme des entités naturelles ${ }^{50}$.Le chapitre de Jeanne Toutous sur la réforme de la carte des régions françaises (chapitre 8) montre bien ces liens entre, d'une part, l'imposition d'un maillage territorial national et d'autre part, des contre-mobilisations de périphéries qui y opposent des cartes alternatives. Votée en 2014 par le Parlement, la réforme de la carte fusionne des régions en France réactive le mouvement régionaliste breton autour de la question du dessin de frontière de la Bretagne, ce dernier revendiquant le rattachement du département de Loire-Atlantique à l'ensemble régional. Par ailleurs, ces « luttes cartographiques »n'illustrent pas simplement une réactivation ent d'u du clivage entre centre et périphérie, mais révèlent également les luttes

\footnotetext{
47 Romain Pasquier, Le pouvoir régional. Mobilisations, décentralisation et gouvernance en France, Paris, Presses de SciencesPo, 2012.

48 Pierre Rosanvallon, Le modèle politique français. La société civile contre le jacobinisme de 1789 à nos jours, Paris, Editions du Seuil.

49 Thiébault Flory, Le mouvement régionaliste français. Sources et développements, Paris, PÜF, 1966.

50 Jacquemine Garel, La construction sociale d'une notion géographique. La notion de groupements régionaux d'après Vidal de la Blache (1880-1940), Thèse pour le doctorat de géographie, Université de Paris 1, 2000.
} 
internes au mouvement régionaliste et portant sur les acteurs légitimes à représenter le territoire breton.

Par ailleurs, dépassant une simple analyse stato-centrée, le présent ouvrage montre comment ce rôle des cartes dans la construction des espaces politiques se transforme du fait de la remise en cause de l'État comme échelle territoriale de référence, à double titre. D’une part, l'émergence, la revendication, et le renforcement de nouvelles échelles de l'autorité politique passent par la stabilisation d'un savoir cartographique sur leurs territoires. La mise en place de représentations homogènes est un des mécanismes de constructions et légitimation, pour les échelles régionales (chapitre 2, Sylvain Le Berre), intercommunale (chapitre 1, Antoine Courmont) et européenne (chapitres 3 de Christine Zanin et 6 de Blandine Mesnel). Analysant les rapports de prospective territoriale, Sylvain Le Berre montre que la mise en image et en carte sert davantage à faire exister une communauté politique territoriale dans le présent qu'à en imaginer le futur, et en marquer les frontières existantes malgré un discours valorisant la trans-territorialité et les interdépendances. Pour les nouvelles entités politiques absorbant une part de souveraineté d'instances préexistantes (telles que l'Union européenne sur les États membres et les intercommunalités sur les communes), les cartes sont un puissant outil d'accompagnement de l'intégration territoriale. La cartographie est mobilisée à Bruxelles comme outil de légitimation de la politique agricole européenne (chapitre 6, Blandine Mesnel). Pour Antoine Courmont, open data et système d'information géographique (SIG) donnent consistance au territoire du Grand Lyon, dépassant les plans et données produits par les services municipaux, et fournissant pour la première fois une représentation unifiée de cette communauté urbaine.

Sur le temps long, le monopole d'Etat sur la production cartographique se discute : comme déjà évoqué précédemment, l'Etat n'a jamais été le seul centre émetteur et producteur de cartes : les Eglises, les marchands, les propriétaires privés, les agents locaux ont toujours plus ou moins contribué à l'élaboration de cartes. Certes, l'Etat a centralisé un certain nombre de prérogatives et de moyens de productions de cartes au cours des guerres du XXème siècle, mais la tendance contemporaine est à une forme de pluralisation des acteurs (et des savoirs) intervenant dans la fabrique des cartes. Or, cette pluralisation en ce début du XXIème siècle n'est pas sans lien avec les reconfigurations territoriales de l'Etat, l'ouverture des modes de gouvernance territoriales et aussi la «transition numérique » de la cartographie ${ }^{51}$ et un ensemble de changements technologiques qui ont affecté durablement le monde de la cartographie et donc des politiques territoriales. Certaines collectivités se sont saisies relativement tôt de nouvelles technologies pour renforcer leur position dans le paysage institutionnel (chapitre 1, Antoine Courmont). A côté de

51 Mark Monmonier, «Introduction », in Mark Monmonsier (eds.), The History of Cartography. Cartography in the 20th Century, Chicago, University of Chicago Press, 2015. 
celles-ci, des acteurs privés (tels que les grandes plateformes Uber, Airbnb et Waze) et des citoyens sont aujourd'hui également producteurs d'un ensemble de données et de cartes, sources d'enrichissement des points de vue, le plus souvent en décalage avec celles issues de la statistique, cartographie et planification officielles (chapitre 4, Boris Mericskay). L'ouvrage invite donc à se saisir de ces nouvelles configurations de production cartographique structurant de plus en plus les pratiques et les représentations des citoyens.

Les cartes contribuent donc à construire les espaces politiques en stabilisant des frontières, en produisant des représentations spatiales et de ce fait en participant à l'institutionnalisation des territoires, du local à l'Europe. Les enjeux et les usages politiques des cartes impliquent donc une perspective multi-niveaux centrée sur les stratégies des acteurs. Il est nécessaire de repérer les institutions, les acteurs, leurs idées, leurs logiques d'action, leurs interactions et d'évaluer les mutations qui affectent les instruments cartographiques. Cela doit permettre de comprendre comment les frontières d'un espace politique se construisent, se stabilisent et changent. Dans un monde interconnecté et traversé par de multiples flux, les frontières prolifèrent de telle manière qu'elles se chevauchent. Les espaces infra-étatiques - régions, métropoles, villes, localités diverses - se présentent comme des espaces emboîtés dont les frontières sont soumises à un processus constant de redéfinition ${ }^{52}$. Les institutions classiques ne sont d'ailleurs qu'un acteur parmi d'autres dans ce processus et il faut donc appréhender l'investissement d'une variété d'acteurs sociopolitiques (partis politiques, mouvements sociaux et culturels, syndicats, ONG) ou économiques (entreprises, réseaux consulaires).

\section{Cartes, représentation des problèmes publics et luttes de représentation}

\subsection{Les cartes dans la construction des problèmes publics}

Comme souligné précédemment, la carte est une forme de savoir et de représentation, renvoyant sous ce rapport à l'étude de la construction des problèmes publics. Les différents chapitres de cet ouvrage le montrent bien, les cartes participent des processus, cognitifs et matériels, d'émergence, d'énonciation et de reformulation des problèmes publics. La production, la circulation et l'usage des cartes cristallisent des représentations particulières des problèmes et des manières de les traiter. Les cartes définissent aussi des publics de l'action publique (soient-ils

52 Romain Pasquier, Vincent Simoulin et Julien Weisbein (dir.), La gouvernance territoriale. Pratiques, discours et théories, Paris, LGDJ, 2013 ( $2^{\text {nd }}$ édition) Martin Vanier, Le pouvoir des territoires - Essai sur l'interterritorialité, Paris, Economica, 2008. 
des espaces ou des populations), en constituant des «publics cibles » et des «publics affectés » ${ }^{53}$, des «gagnants» et des «perdants ». Elles deviennent de ce fait des objets de mobilisations collectives et de conflits. La dimension spatiale propre aux représentations cartographiques est centrale pour en comprendre les enjeux dans la construction de l'action publique. Elle renvoie aux questions de distance et de proximité, aux limites d'inclusion et exclusion, aux centralités et hiérarchies, aux dynamiques et aux effets de localisation des acteurs sociaux. Elle concerne des objets matériels (infrastructure, ville...), immatériels (flux d'information, frontières...) ou idéels (conflits, mobilisations...). ${ }^{54}$ Ces dimensions spatiales des problèmes publics sont souvent présentes dans le débat public, par exemple lorsque des attentes sociales sur l'aménagement des territoires et sur la qualité environnementale sont confrontés aux logiques gestionnaires et financières de l'action publique, avec une tension entre expectatives et contraintes, demande et offre, notamment dans les cartes d'accès aux services (chapitre 13 de Clara Deville).

Les problèmes publics sont des construits socio-politiques. Leur émergence se fait par la qualification d'une situation comme anormale (étape d'identification), l'implication d'entrepreneurs de cause (qui peuvent être des élites de l'administration ou du monde politique, ou mouvements sociaux) (étape de définition) et la mise en débat et la prise en charge par des autorités publiques (étape de publicisation) $)^{55}$, tant dans des arènes publiques ${ }^{56}$ que dans des instances administratives plus «discrètes ${ }^{57}$ Or, la qualification de la situation perçue ou reconnue comme anormale peut en partie reposer sur des approches de spatialisation et de cartographie (montrant par exemple des nuisances environnementales, des inégalités de répartition des équipements scolaires ou d'accès aux soins, etc.). La cartographie peut mettre au jour des phénomènes de différenciations socio-économiques dans l'espace ou d'inégalités d'accès aux équipements. Des « entrepreneurs de cause » peuvent s'en saisir pour façonner ou stabiliser des représentations du problème public. De même, la mise à l'agenda par les autorités publiques peut aussi s'accompagner d'une production de cartes, par exemple dans le cadre des projets urbains d'urbanisme et d'équipement (plans, schémas, cartes de localisation...). Les débats précurseurs du

\footnotetext{
${ }^{53}$ Peter Knoepfel, Corinne Larrue, Jean-François Savard, Frédéric Varone, Analyse et pilotage des politiques publiques. France, Suisse, Canada, Presses de l'Université du Québec, 2015 ; Lorenzo Barrault-Stella, PierreÉdouard Weill (eds.), Creating target publics for welfare policies. A comparative and multilevel approach, Springer International Publishing, « Logic, argumentation and reasoning », 2018.
}

54 Jacques Lévy et Michel Lussault, « Espace », dans Lévy J. et Lussault M. (dir.), Dictionnaire de la géographie et de l'espace des sociétés, Belin, 2003, p.325-333.

55 Elizabeth Sheppard, "Problème public », in L. Boussaguet et al., Dictionnaire des politiques publiques, Presses de Sciences Po, 2010, p.530-538.

56 Daniel Cefaï, «La construction des problèmes publics. Définitions de situations dans des arènes publiques », Réseaux, vol. 14, $\mathrm{n}^{\circ}$ 75, 1996, p. 43-66.

57 Claude Gilbert et Emmanuel Henry, «La définition des problèmes publics : entre publicité et discrétion », Revue française de sociologie, vol. 53, ${ }^{\circ} 1,2012$, p. 35-59. 
projet du Grand Paris Express dans les années 2005-2010 ont donné lieu à une production régulière de cartes, d'autant plus qu'ils se doublaient d'une confrontation entre acteurs publics de différents échelons territoriaux défendant des logiques et tracés distincts. Outils bien connus par les administrations locales, les cartes peuvent également être mobilisées pour redéfinir localement les limites et les bénéficiaires des politiques nationales. Les négociations par l'intercommunalité de Plaine Commune autour du zonage étatique de la nouvelle géographie prioritaire analysées dans cet ouvrage par Violette Arnoulet en constituent une illustration éclairante (chapitre 10). Cet établissement public de coopération intercommunale (EPCI), très outillé en savoirs et outils cartographiques, engage un véritable débat cartographique avec les services de l'Etat, pendant lequel la représentation spatialisée d'indicateurs est mobilisée pour revendiquer la nécessité d'une appréciation locale des périmètres et du problème à traiter.

Les cartes ont un pouvoir de définition des problèmes publics (chapitre 7 de Nicolas Larchet) par leurs effets de connaissance et méconnaissance de l'objet représenté tout comme des modalités de représentation retenues. Elles sont donc un «marqueur concret» du cadrage des problèmes publics et ses évolutions, mais sont également des institutions contribuant à orienter les comportements des acteurs impliqués. Dans cette perspective, le chapitre de Nicolas Larchet montre que le problème public de l'accès à l'alimentation des plus pauvres fait l'objet de recadrages d'une carte à l'autre (et d'une ville à l'autre) aux États-Unis : le problème est défini en termes de lutte contre la faim, puis de sécurité alimentaire des communautés et enfin d'obésité. Chacune de ces définitions repose sur des interprétations différentes de l'origine du problème (inégalités socio-économiques structurelles ou simple problème d'offre et demande d'aliments "sains" ?) et fait appel à des types de solutions également différenciées. Par ailleurs, ce pouvoir de définition porté par les cartes et les zonages peut devenir lui-même l'objet de critiques. C'est ce que montre Renaud Epstein dans cet ouvrage (chapitre 12). A l'aune du travail d'Albert Hirschman sur la rhétorique réactionnaire, l'auteur retrace le succès de la thèse des effets pervers (enfermement, discrimination, stigmatisation) créés par le zonage des « quartiers prioritaires » qui en font des objets spécifiques d'action publique. Cette thèse de l'effet pervers est largement mobilisée dans le débat politique et savant pour montrer l'inefficacité d'une politique publique dont la légitimité est déjà fortement remise en cause.

Les usages politiques des cartes ne se réduisent pas à la construction et la définition des problèmes publics : les cartes sont mobilisées pour légitimer des décisions et des programmes de politiques publiques autour de ces problèmes. Par exemple, du côté des administrations et du monde politique, le recours à la représentation cartographique, interactive notamment, peut être utilisée pour appuyer le caractère désirable des mutations spatiales urbaines, de concert avec l'offre de simulations 3D des espaces proposées pour «donner à voir » l'aspect du lieu une fois 
construit/transformé. L'usage de l'image au sens large dans la communication urbaine, le projet urbain et la participation a fait l'objet de travaux déjà consolidés. ${ }^{58}$ Dans cet ouvrage, Blandine Mesnel montre que la cartographie agricole tire aussi son origine du souci des fonctionnaires de la Commission européenne de renforcer la légitimité de la Politique Agricole Commune afin d'en préserver le budget (chapitre 6). Dans leurs attentes, la cartographie doit permettre d'améliorer l'efficacité budgétaire et la transparence des procédures, et atteindre des objectifs de préservation de l'environnement pour lesquels elle est fortement critiquée. De même, pour le cas de la politique de la ville, un instrument d'exploitation cartographique et statistique (le carroyage), participe de la construction de la légitimité de la refonte du zonage. Cet instrument incorpore dans son design et technicité des négociations politiques et des stratégies pour faciliter son acceptation. Il internalise notamment l'objectif de réduire le nombre de quartiers de la politique de la ville tout en maintenant ses territoires historiques. Une analyse des cartes dans la définition et formulation des problèmes publics ne peut donc être dissociée de l'étude des propriétés sociales des leurs producteurs, des rapports de forces entre les acteurs impliqués, ainsi que d'une archéologie des sources utilisées. La cartographie elle-même peut être issue de bases de données publiques, mais peut aussi émaner de la production de chercheurs et ingénieurs des laboratoires publics, d'experts consacrés par la sphère médiatique, mais encore de la participation des publics concernés ou de groupes militants. Contrairement aux travaux qui considèrent les cartes comme un instrument exclusif des gouvernants, plusieurs chapitres de cet ouvrage montrent que les usages politiques des cartes par les gouvernés jouent un rôle central dans l'action publique.

\subsection{La carte, vecteur et support de résistances à l'action publique}

Pour le géographe Brian Harley, l'histoire de la cartographie met au jour une forme d'inégalité : « les flèches idéologiques ont été largement tirées dans un seul sens, au sein de la société, des puissants vers les faibles. À la différence de la littérature, de l'art ou de la musique, l'histoire sociale des cartes ne paraît pas comporter des modes d'expression populaires, alternatifs ou subversifs ${ }^{59}$. Les cartes sont essentiellement un langage de pouvoir et non de contestation ». Il ajoutait que «la technologie informatique a même renforcé cette concentration du pouvoir des médias ». Au contraire, les chapitres réunis ici apportent trois bonnes raisons de s'opposer à cette vision et de battre en brèche l'idée d'un monopole d'État sur la conception et l'usage de la cartographie exposée ici par Brian Harley.

58 Hélène Bailleul, «Les nouvelles formes de la communication autour des projets urbains : modalités, impacts, enjeux pour un débat participatif », Métropoles [En ligne], $\mathrm{n}^{\circ} 3, \quad 2008$, URL : http://journals.openedition.org/metropoles/2202.

59 Brian Harley, « Maps, knowledge and Power », art.cit., p. 48. 
Premièrement, il s'agit de ne pas surestimer le pouvoir des cartes de la même manière qu'il ne faut pas surestimer le pouvoir de la statistique. Si les démographes, les sociologues et les politistes ont eu tendance à surestimer le poids de la production de connaissance et d'expertise dans la conception des politiques publiques ${ }^{60}$, les géographes ont aussi eu tendance à faire de la carte l'alpha et l'oméga de la conception de politiques territoriales éclairées. Soutenir qu'il faut connaitre spatialement les pratiques et représentations des groupes sociaux pour aménager les territoires de façon plus pertinente est assurément un préalable, mais à y regarder de plus près, rares sont les exemples de politiques publiques qui présentent des processus aussi linéaires. Ainsi, à l'encontre d'une équation foucaldienne « savoir = pouvoir » trop symétrique et qui surestime le poids du savoir pour la conquête et l'exercice du pouvoir, certains travaux ont montré que ce n'est pas le contenu des bases de données et des cartes qui soutient l'acquisition de pouvoir mais bien la ressource organisationnelle qu'ils représentent dans des champs d'acteurs conflictuels ${ }^{61}$. La carte en soi n'est pas une ressource pour gouverner, mais c'est la croyance en ses vertus qui assure à ses concepteurs une légitimité à le faire. Pour le dire de façon plus triviale, les administrations ont beau produire de plus en plus de données et de cartes aussi fines soient-elles, les décideurs ne s'en servent que rarement, en tout cas moins que ce que les chercheurs en sciences sociales le supposent.

Deuxièmement, comme c'est le cas pour tout instrument ${ }^{62}$, les cartes rencontrent des résistances dans la conception, la mise en œuvre et l'appropriation. Elles peuvent être détournées (servir des intérêts divergents par rapports aux supposés objectifs initiaux de décideurs), contournées (singer une application tout en s'en défiant) ou neutralisées (des stratégies peuvent être développées pour empêcher la mise en œuvre et la production d'effets). Une grande partie de cet ouvrage met bien en évidence la diversité des formes de résistances dans différentes séquences du processus de cartographie et d'institutionnalisation des politiques publiques. Par exemple, Blandine Mesnel montre bien que la carte ne fait pas consensus et suscite de vifs conflits entre des services et des administrations, avant même de rencontrer des résistances de la part des cibles (chapitre 6). C'est le cas également pour la

60 Et à l'inverse, on peut renvoyer aux travaux insistant plutôt sur le rôle de l'ignorance dans l'action ou l'inaction publique : Emmanuel Henry, Ignorance scientifique et inaction publique, Paris, Presses de Sciences Po, 2017.

61 Theodore Porter, Trust in Numbers. The Pursuit of Objectivity in Science and Public Life, Princeton, Princeton University Press, 1995.

62 Pierre Lascoumes et Jean-Pierre Le Bourhis, «Les résistances aux instruments de gouvernement. Essai d'inventaire et de typologie des pratiques », in Charlotte Halpern, Pierre Lascoumes et Patrick Le Galès, L'instrumentation de l'action publique, Paris, Presses de Sciences Po, 2014, p. 493-520. 
nouvelle carte des régions françaises en prise aux mobilisations régionalistes comme le montre Jeanne Toutous (chapitre 8). Violette Arnoulet analyse quant à elle les résistances des collectivités locales face à la nouvelle géographie prioritaire lancée par l'État (chapitre 10). Dans le même domaine, Mauricio Aranda et Juliette Froger-Lefebvre mettent en évidence la façon dont des agents (des militantes) bricolent et ajustent la mise en œuvre de programmes dans un climat à la fois de contrainte et d'incertitude (chapitre 9). Enfin, la carte liée à des réformes (notamment de politiques constitutives et de réorganisation de l'État sur les territoires) suscite voire arme des conflits. C'est ce qui a été montré par Lorenzo BarraultStella et Francesca Artioli dans leurs travaux respectifs sur la carte scolaire et la carte militaire. Tous ces exemples rappellent que la carte n'est pas un instrument de domination et de pacification à sens unique, qu'elle est aussi support de résistance politique forte.

Troisièmement, les cartes ne relèvent pas d'un monopole des élites, ni des experts ou des géographes travaillant pour l'État. La cartographie, comme la statistique, a pu trouver une partie de ses origines dans des organisations non étatiques comme les paroisses ${ }^{63}$ ou des entreprises privées. De plus, là aussi comme la statistique, la cartographie a toujours été une ressource pour les individus ou les groupes pour résister aux politiques publiques mais aussi aux acteurs de marchés, aux propriétaires fonciers. En opposant aux savoirs d'État ou d'experts des cartographies alternatives, des citoyens peuvent résister à des expulsions de logement ${ }^{64}$, dénoncer des «projets inutiles» et proposer d'autres façons d'aménager le territoire $^{65}$, inviter à occuper des espaces vacants dans les villes ${ }^{66}$. A l'instar d'Isabelle Bruno et d'Emmanuel Didier qui parlent de « statactivisme ${ }^{67}$, il est possible de parler de « cartoactivisme». Ainsi, en soi, la carte devient un instrument d'action collective ou de mobilisation citoyenne quand elle est créée, mise à disposition et publicisée dans le cadre de la construction des problèmes publics, de leur mise à l'agenda ou de conflits autour de politiques existantes. La tonalité de cette mobilisation peut parfois être participative, par exemple dans le cadre des citoyens référents ou sentinelles qui collectent de la donnée pour

63 Florian Mazel, L'évêque et le territoire. L'invention médiévale de l'espace (Vème-XIIIème siècle), Paris, Le Seuil, 2016.

64 Arjun Appadurai, «Why enumeration counts », in Environment and Urbanization, vol. 24, n², 2012, p. 1-3. 65 Collectif «Les plumes dans le goudron», Résister aux grands projets inutiles et imposés. De Notre-Damedes-Landes à Bure, Editions Textuel, 2018.

66 On pense par exemple à toutes les expériences de cartographie des logements vacants dans les années 2011 à la suite des mouvements des Indignés en Espagne, Occupy Wall Street aux États-Unis, mais aussi en France où certains collectifs de squatteurs ont constitué des bases de données et des cartes des logements vacants à Paris (cf. Thomas Aguilera, «DAL et Jeudi Noir, deux usages du squat dans la lutte contre le mal-logement », in Métropolitiques, 25/04/2012).

67 Isabelle Bruno, Emmanuel Didier, Julien Prévieux, Statactivisme. Comment lutter avec des nombres, Paris, La Découverte, 2014. 
les collectivités ou associations (par exemple sur la qualité de l'air) voire pour des suivis scientifiques pilotés par des chercheurs (par exemple, les observatoires de Vigie-Nature du Muséum national d'Histoire naturelle). D'ailleurs, à rebours de ce qu'avançait Brian Harley, les avancées technologiques notamment liées au développement d'Internet et du big data ont en partie démocratisé la conception et l'usage des cartes en donnant aux citoyens la possibilité de se saisir d'éléments inaccessibles jusqu'alors. Cette «démocratisation» des objets connectés et des applications qu'elles contiennent démultiplient simultanément la possibilité de recueils de données et de diffusion de cartes. Les SIG (Systèmes d'Information géographique) permettent de superposer les données et de les combiner. Ils requièrent certes une technicité et des compétences spécifiques, mais les groupes peuvent trouver en leur sein ou développer ces compétences. Boris Merisckay le montre bien dans le chapitre 4 consacré au lien entre cartographie, gouvernance et données territoriales, le développement de plateformes de conception cartographique participative a permis à des citoyens ou à des groupes de porter des revendications auprès des décideurs mais aussi de participer à la mise en œuvre de l'action publique voire d'interventions citoyennes alternatives aux politiques publiques.

Néanmoins, il est important de souligner que ce lien entre changement technologique et « démocratisation » est complexe et n'est pas monodirectionnel. Comme il a été montré par les travaux sur les politiques de la «ville intelligente », la production et la construction de systèmes d'information cartographiques peut être ouverte, collaborative et décentralisée mais également fermée et monopolistique, y compris dans les mains d'acteurs privés ${ }^{68}$. Cela pose la question sousjacente de la donnée, et des conditions de son accessibilité et circulation. En effet, si les avancées technologiques ont entrainé une démultiplication de données spatialisées, une partie d'entre elles est fermement dans les mains d'entreprises, en premier les géants du numérique, en faisant un usage opaque et peu, ou pas, accessible. Cela pose la question sous-jacente de la valeur de la donnée, et de celle qui est reconnue comme légitime par les acteurs sociaux et par les pouvoirs institués en charge du problème public.

\section{Cartes, inertie et changements dans l'action publique}

68 Dominique Boullier, «Politiques des villes intelligentes» in Actes du colloque Ville intelligente, ville démocratique ?, Berger-Levrault, Chaire MADP Sciences Po, Février 2014 ; Antoine Courmont et Patrick Le Galès, Gouverner la ville numérique, Paris, Presses Universitaires de France, 2019. 


\subsection{Usages réformateurs et inertie des cartes dans l'action publique}

En complément des aspects liés à la représentation des problèmes publics véhiculée par la cartographie, d'autres dimensions sont susceptibles de générer des conflits. Comme toute institution ou instrument d'action publique, les cartes, bien que dotées de caractéristiques intrinsèques potentiellement contraignantes et génératrices d'inertie, existent avant tout par leurs usages sociaux et politiques. Les usages réformateurs des cartes peuvent parfois permettre d'impulser des changements de grande ampleur, toujours dépendants des structures institutionnelles $^{69}$, dans un secteur ou un autre d'action publique ${ }^{70}$. L'institutionnalisation et les ajustements de la carte scolaire au début des années 1960 en France s'inscrivent par exemple dans un mouvement plus général de mise à plat du système d'enseignement et de préparation bureaucratique de la «démocratisation» de l'institution scolaire qui sera réalisée à la fin des années $1970^{71}$. Les réformes de la carte militaire entreprises depuis 2008 relèvent du mouvement plus général de création de «pôles» de services administratifs, justifié au nom de la rationalisation des dépenses et d'une plus grande efficacité qui viendrait de la mutualisation ${ }^{72}$. Toutefois, dans nombre de cas, les cartes présentent une grande inertie et leur actualisation politique nécessite de surmonter diverses résistances institutionnelles, syndicales ou autres. Tel est par exemple le cas de la réforme de la carte judiciaire en France ${ }^{73}$ ou encore des ajustements, initiés dès 2002 et aboutis seulement en 2014, de cette même carte judiciaire en Belgique ${ }^{74}$.

Cette inertie tendancielle de l'instrument cartographique, partiellement liée à des formes bien connues de dépendance au sentier ${ }^{75}$, conduit souvent les gouvernants à mettre en scène une réforme et à jouer de la politique symbolique ${ }^{76}$ sans introduire de modifications substantielles

69 Wolfgang Streek, Kathleen Thelen, Beyond Continuity. Institutional Change in Advanced Political Economy, Oxford, OUP, 2005 ; Anne-France Taiclet, «La professionnalisation des développeurs territoriaux comme mécanisme de production de normes dans l'action publique », in Flora Bajard, Bérénice Crunel, Caroline Frau, Frédéric Nicolas, Fanny Parent (dirs.), Professionnalisation(s) et État: une sociologie politique des groupes professionnels, Villeneuve-d'Ascq, France, Presses universitaires du Septentrion, 2018.

70 Jacques Fontaine, Patrick Hassenteufel (dir.), To change or not to change? Les changements de l'action publique à l'épreuve du terrain, Rennes, PUR, 2005.

71 Lorenzo Barrault-Stella, Gouverner par accommodements. Stratégies autour de la carte scolaire, Paris, Dalloz, 2013, p.109-147.

72 Francesca Artioli, «Les politiques du retrait territorial de l'État. Réformes de la carte militaire et gestion des mobilisations locales (1989-2012)», Gouvernement et action publique, n 1, 2017, p. 81-106.

73 Jacques Commaille, Territoires de justice. Une sociologie politique de la carte judiciaire, Paris, PUF, 2000 ; Étienne Cahu, « De la réforme de la carte judiciaire ou l'instrumentalisation de critères objectifs au service d'un nouveau rapport scalaire de pouvoir », Annales de géographie, $\mathrm{n}^{\circ} 701,2012, \mathrm{p} .5-30$.

74 Joël Ficet, «Trajectoires de réforme de la carte judiciaire et managérialisation de l'État. Analyse comparée des politiques de territorialisation de la Justice en France et en Belgique », Revue internationale de politique comparée, vol.18, n4, 2011, p.91-118.

${ }^{75}$ Paul Pierson, «Increasing returns, path dependence, and the study of politics », American political science review, vol. $94, \mathrm{n}^{\circ} 2,2000$, p. 251-267.

${ }^{76}$ Murray Edelman, The symbolic uses of politics, Chicago, University of Illinois Press, 1964. 
dans l'action publique. Le volontarisme politique de N. Sarkozy en 2007 en matière de carte scolaire en constitue une illustration ${ }^{77}$. Il arrive même que l'affichage de la stabilité d'une carte d'action publique autorise l'euphémisation de certains phénomènes politiquement dérangeants à l'image, comme c'est le cas pour la carte de l'eau au Brésil ${ }^{78}$ ou encore concernant la cartographie différentielle du logement informel en Europe ${ }^{79}$ : l'association de formes d'inaction publique et d'une stratégie de communication contribue ainsi à l'invisibilisation de certains problèmes sociaux (voir entre autres le chapitre 11 proposé par Kévin de la Croix et Nicolas Verhaeghe quant à la cartographie des cours d'eau), évitant l'émergence de catégories raciales dans les milieux étatiques comme dans les débats publics ${ }^{80}$, ou à l'inverse au recadrage d'enjeux sociaux en enjeux ethno-raciaux ${ }^{81}$. Dans tous les cas, par ses effets d'objectivation voire de naturalisation (ou à l'inverse d'occultation), les cartes sont partie prenante du travail politique de légitimation (ou de déni) $^{82}$, comme l'illustre par exemple les cartes liées aux politiques de peuplement ${ }^{83}$ ou encore les cartes prioritaires de santé étudiées par Mauricio Aranda et Juliette Froger-Lefebvre dans cet ouvrage (chapitre 9).Ces usages réformateurs, potentiellement très diversifiés et souvent imprévisibles du côté des institutions comme des gouvernés ${ }^{84}$, peuvent par ailleurs entrainer des transformations même de l'instrument. En attestent les évolutions de la carte du bruit à Paris ${ }^{85}$, des cartes des politiques de la ville ${ }^{86}$ ou encore de celles des zones inondables dans le sud de la

77 Lorenzo Barrault-Stella, «Les résistances de la carte scolaire. Les limites du volontarisme politique dans l'action publique », Politix, ${ }^{\circ} 98,2012$, p. 109-128.

78 Gilles Massardier, Suyá Quintslr, «Explaining path dependance and blame avoidance : policy coalitions and water plan in Duque de Caxias City 2007-2016 (RJ, Brazil) », in Franck Poupeau et ali. (eds.), Water conflicts and hydrocracy in the Americas : coalitions, networks, policies, São Paulo, Brésil, IEE-USP, 2018, p. 89-120.

79 Thomas Aguilera, «L'informalité urbaine aux marges de la connaissance. Statistiques, cartographie et politique des bidonvilles à Madrid », in Anthropologica, 2019, p. 35-50.

80 Dvora Yanow, Constructing «Race » and «Ethnicity» in America. Category Making in Public Policy and Administration, London, Sharpe, 2003.

81 Eric Fassin, Carine Fouteau, Serge Guichard, Aurélie Wendell, Roms et riverains. La politique municipale de la race, Paris, La Fabrique, 2014.

82 Que l'on peut classiquement définir « comme un ensemble de processus qui rendent l'existence d'un pouvoir coercitif spécialisé tolérable, sinon désirable, c'est-à-dire qui le fasse concevoir comme une nécessité sociale, voire comme un bienfait »: Jacques Lagroye, «La légitimation », Traité de science politique, PUF, Volume 1, 1985, p. 402.

83 Fabien Desage, Christelle Morel-Journel, Valérie Sala-Pala (dir.), Le peuplement comme politiques, Rennes, PUR, 2014.

84 Lorenzo Barrault-Stella, « Jouer avec l'instrument. Les usages institutionnels et sociaux de la carte scolaire », in Charlotte Halpern, Pierre Lascoumes, Patrick Le Galès (dir.), L'instrumentation de l'action publique. Controverses, résistances, effets, Paris, Presses de Sciences Po, 2014, p. 397-415. Un exemple récent en est aussi fourni par la communication ministérielle de données relatives aux choix universitaires dans Parcoursup, qui a pu donner lieu à la construction militante de cartes visant à critiquer le dispositif : voir par exemple https://cartonumerique.blogspot.com/2018/05/que-vaut-la-datamap-qui-geolocalise-les.html?m=1 (consulté le 16 mai 2019).

85 Philippe Zittoun, «La carte parisienne du bruit. La fabrique d'un nouvel énoncé de politique publique », Politix, n`78, 2007, p. 157-178.

86 Philippe Estèbe «Les quartiers, une affaire d'État », op cit. ; Sylvie Tissot, L'État et les quartiers. Genèse d'une catégorie de l'action publique, Paris, Seuil, 2007. 
France $^{87}$. L'adjonction de dérogations à la carte scolaire dont les usages (institutionnels à des fins gestionnaires, comme par les familles les mieux informées) croissants depuis les années 1980 ont largement redéfini l'instrument. Rares sont les cas de reconfigurations fulgurantes : les effets de transformation de l'instrument par ses usages se font généralement à petits pas, de façon incrémentale, parfois même sans avoir été pensés politiquement. Les institutions, dans lesquelles évoluent de multiples acteurs aux intérêts variés, sont pesantes, ne laissant bien souvent la marge qu'à des «changements graduels transformateurs ${ }^{88}$. Les luttes autour du sens et des usages de l'instrument sont inextricablement mêlées aux allocations de ressources que l'actualisation de la carte conditionne. Au-delà de la grande variabilité des usages politiques et sociaux de l'instrument, l'une des régularités observables dans divers pays occidentaux depuis une quarantaine d'années et ce que certains auteurs ont nommé le «tournant néo-libéral » ${ }^{89}$ est l'inscription des réformes cartographiques dans divers secteurs d'action publique au cœur des recompositions de l'État.

\subsection{Retrait et recompositions des cartes de l'État}

Dans la période contemporaine, les mutations des cartes de l'action publique sont dans bien des cas à la fois l'enjeu et le produit des reconfigurations de l'État, des institutions publiques et, par cette médiation, des relations gouvernants-gouvernés. Les cartes renvoient en effet à l'organisation spatiale des services publics et des administrations (cartes judiciaire, scolaire, militaire, des collectivités locales, etc.). Elles constituent ainsi les instruments des politiques constitutives en ce qu'elles participent aux processus de réformes internes aux États et portant sur ses structures. On peut dans cette optique étudier les transformations des cartes de l'action publique - au sens des mutations spatiales et organisationnelles des services publics et des administrations -, ainsi que les effets de ces réformes sur la mise en œuvre de l'action publique et, plus largement, sur les groupes sociaux et professionnels qui y sont confrontés. Les recompositions en cours des États, en Europe mais pas uniquement, entremêlent des dynamiques internes aux structures étatiques (les politiques de réformes de l'administration notamment) et des logiques externes liés aux relations avec d'autres institutions publiques à différentes échelles de gouvernement (l'Union européenne ou les

87 Jean-Pierre Le Bourhis, « Du savoir cartographique au pouvoir bureaucratique », op cit.

88 James Mahoney, Kathleen Thelen, «A Theory of Gradual Institutional Change » in James Mahoney, Kathleen Thelen (eds), Explaining Institutional Change. Ambiguity, Agency and Power, Cambridge, Cambridge University Press, 2010, p. 1-39.

89 Bruno Jobert, Bruno Theret, «France : la consécration républicaine du néo-libéralisme », in Bruno Jobert (dir.), Le tournant néo-libéral en Europe, Paris, L’Harmattan, 1994, p. 21-85. 
collectivités locales) et privées (les mondes économiques). Ces reconfigurations sont pour partie objectivées par les mutations de diverses cartes des institutions publiques ${ }^{90}$. La distribution socio-spatiale des administrations et des services publics s'en trouve affectée et, dans divers pays occidentaux, on observe des formes de retrait ou de rétraction ${ }^{91}$ voire de «démantèlement » de l'État au sein des territoires ${ }^{92}$. C'est par exemple le cas de la carte judiciaire, aussi bien en France qu'en Italie ou en Belgique, où des logiques gestionnaires ont conduit à des regroupements et à diverses fermetures de petites juridictions ${ }^{93}$. Cette tendance à la concentration et à la restriction est la même dans divers secteurs d'action publique, à l'instar des politiques hospitalières ${ }^{94}$ (entre autres par les fermetures d'hôpitaux ruraux et petites maternités), des mutations des services postaux ${ }^{95}$, des transports ${ }^{96}$, des forces armées ${ }^{97}$ ou des universités ${ }^{98}$. À chaque fois, les transformations de la carte du service concerné réfractent les recompositions institutionnelles et étatiques en cours. Or, ces recompositions des institutions publiques et de l'État sont sensibles pour les populations dont la proximité et l'égalité d'accès aux services publics est parfois remise en cause ${ }^{99}$. L'étude proposée dans cet ouvrage par Patrice Caro, Agnès Checcaglini et Arnaud Lepetit quant à l'accessibilité des établissements scolaires en est une illustration (chapitre 14), tout comme l'enquête de Clara Deville sur l'opérationnalisation du RSA au prisme des expériences de ses demandeurs (chapitre 13). Dans bien des cas, les reconfigurations des cartes suscitent des résistances voire des mobilisations (localisées, par les professionnels du secteur, des groupes d'usagers, des syndicats, etc.), dont les acteurs produisent parfois des données alternatives, conçoivent et mobilisent une contre-cartographie pour s'opposer à l'expertise d'État et tenter d'infléchir ses réformes. Face à ces risques de conflit et de sanction politique ou électorale à leur encontre,

90 Dans le même sens : Patrick Hassenteufel, « L’État mis à nu par les politiques publiques », in Bertrand Badie, Yves Déloye (dir.), Le temps de l'État. Mélanges en l'honneur de Pierre Birnbaum, Paris, Fayard, 2007, p. 311329.

91 Neil Brenner, New State Spaces: Urban Governance and the Rescaling of Statehood, Oxford, Oxford University Press, 2004.

92 Paul Pierson, Dismantling the Welfare State? Reagan, Thatcher and the Politics of Retrenchment, Cambridge, Cambridge University Press, 1995.

93 Cécile Vigour, Réformes de la justice en Europe, Bruxelles, De Boeck, 2018, p. 261.

94 Pierre-André Juven, Frédéric Pierru, Fanny Vincent, La casse du siècle. À propos des réformes de l’hôpital public, Paris, Raisons d'agir, 2019.

95 Nadège Vézinat, Les métamorphoses de la Poste (1953-2010), Paris, PUF, 2012.

96 Laurent Kestel, En Marche forcée. Une chronique de la libéralisation des transports : SNCF, cars Macron et quelques autres, Paris, Éditions Raisons d'agir, 2018.

97 Francesca Artioli, «Les politiques du retrait territorial de l'État. Réformes de la carte militaire et gestion des mobilisations locales (1989-2012)», Gouvernement et action publique, n¹, 2017, p. 81-106.

98 Christine Musselin, La grande course des universités, Paris, Presses de Sciences Po, 2017.

99 Jean-Marc Weller, «Le travail administratif, le droit et le principe de proximité », L'Année sociologique, vol. 53, n², 2003, p. 431-458; Clara Deville, «Les chemins du droit. Ethnographie des parcours d'accès au RSA en milieu rural », Gouvernement et action publique, $\mathrm{n}^{\circ} 3,2018, \mathrm{p} .83-112$. 
les élus nationaux et locaux déploient alors des stratégies d'évitement du blâme ${ }^{100}$ en mobilisant des politiques symboliques ou en intégrant dans les politiques de réformes de l'État de modalités de gestion ou de domestication des résistances. En France, la gestion des mobilisations locales face aux fermetures de casernes est par exemple un enjeu ministériel ${ }^{101}$. De même, après plusieurs décennies de tensions autour des fermetures scolaires, le Ministère de l'Éducation nationale comme les administrations déconcentrées prennent le plus grand soin pour produire des réformes socialement acceptables au sein des territoires en cas de suppression de classes ou d'établissements ${ }^{102}$. Les cartes des services publics sont ainsi des enjeux centraux à la fois dans les mutations institutionnelles et dans les transformations des relations gouvernants-gouvernés. Dans un contexte historique de crise économique, les recompositions étatiques et des administrations objectivées par les mutations cartographiques sont susceptibles de produire des effets de contraintes forts pour les usagers, voire conduisent parfois à un accroissement des inégalités sociales et territoriales face à l'État et à l'allocation de ressources publiques ${ }^{103}$. C'est d'ailleurs ce que suggère l'enquête restituée dans l'ouvrage par Magali Nonjon, Julien O'Miel et Julien Talpin quant à la refonte de la géographie prioritaire en 2014 (chapitre 5). Ces phénomènes s'inscrivent dans des questionnements classiques autour de la gouvernabilité et des capacités d'action du politique ${ }^{104}$ et il n'y a rien d'étonnant à ce que les gouvernants s'interrogent sur les conséquences politiques (notamment électorales ${ }^{105}$ ) de ces projets du point de vue des gouvernés. Cet objet permet de questionner le poids des luttes intra et inter-institutionnelles dans les réformes étatiques et de discuter l'hypothèse d'une accentuation des inégalités socio-spatiales face aux administrations et services publics.

100 R. Kent Weaver, «The Politics of Blame Avoidance », Journal of Public Policy, 654), 1986, p. 371-398 ; Christopher Hood, The blame game. Spin, bureaucracy and self-preservation in government, Princeton, Princeton University Press, 2011.

101 Francesca Artioli, «Les politiques du retrait territorial de l'État. Réformes de la carte militaire et gestion des mobilisations locales (1989-2012) », Gouvernement et action publique, n 1, 2017, p. 81-106.

102 Lorenzo Barrault-Stella, « Produire un retrait de l'État acceptable. Les politiques de fermetures scolaires dans les mondes ruraux contemporains », Gouvernement et action publique, n³, 2016, p. 33-58.

103 Voir entre autres Sylvie Tissot, Franck Poupeau, «La spatialisation des problèmes sociaux », Actes de la recherche en sciences sociales, $\mathrm{n}^{\circ} 159,2005$.

${ }^{104}$ Renate Mayntz, «Governing failures and the problem of governability », dans J. Kooiman (éds), Modern governance, New government-society interaction, Sage, 1993, p. 9-20.

105 Natalie Giger, Moira Nelson, «The Electoral Consequences of Welfare State Retrenchment : Blame Avoidance or Credit Claiming in the Era of Permanent Austerity ? », European Journal of Politicial Research, 50,2010 , p. 1-23. Les prolongements de ces questionnements ont donné lieu à de nombreux travaux relatifs aux policy feedback. 


\section{La construction de l'ouvrage}

Parce qu'elles s'inscrivent à la croisée de recompositions politiques, de mutations territoriales et de transformations des liens entre gouvernants et gouvernés, les cartes de l'action publique constituent aujourd'hui un objet central en géographie, en science politique et en sociologie, justifiant largement une problématisation pluridisciplinaire voire interdisciplinaire. C'est donc dans cette perspective que cet ouvrage interroge la façon dont les changements des cartes transforment les modes de gouvernement des populations et des territoires locaux, et comment ces derniers contribuent (ou non) à donner du sens à ces découpages et contenus territoriaux, à les utiliser, à les combattre voire à les dépasser. Cette perspective s'intéresse alors aux effets des réformes sur les liens gouvernants-gouvernés, dans les processus de légitimation de l'action publique et sur la prise en compte des diverses formes d'inégalités sociales et territoriales. Mais, en lien nécessaire, l'ouvrage revient également sur les conditions de production des cartes et leurs effets sur les mécanismes politiques qui s'en suivent. Loin de mettre à distance la technicité de ces instruments, il s'agit de s'en saisir afin d'isoler les effets propres des outils et des techniques qui les incarnent tout en les insérant dans les jeux d'acteurs qui les mobilisent et les exploitent.

Ces changements sont étudiés dans l'ouvrage à travers une perspective multiniveaux et par la confrontation entre différents secteurs d'action publique (politiques de la ville, de gestion de l'eau, d'éducation, d'aménagement du territoire, de santé et politiques de réforme administrative). Basés sur des matériaux empiriques robustes et inédits, les chapitres relèvent de plusieurs champs disciplinaires en sciences sociales (science politique, sociologie, géographie et aménagement). L'ouvrage, comme le colloque dont il est pour une part issu, fait le pari de la pluridisciplinarité : non pour suivre une injonction du temps, non pas seulement parce que la carte est prise comme objet par diverses disciplines, mais surtout parce cela a permis de générer de nouvelles hypothèses et de produire de nouvelles interprétations sur le rôle des cartes dans l'action publique aujourd'hui.

Dans ces perspectives, l'ouvrage est organisé en quatre parties qui éclairent les cartes de l'action publique dans leurs liens avec l'institutionnalisation, le changement, la mise en œuvre et le traitement des inégalités socio-spatiales par l'action publique. La première partie analyse les liens entre la construction d'un savoir cartographique et la consolidation d'une autorité politique. Au-delà de l'État nation qui a été l'objet privilégié de ces questionnements, les liens entre représentation, institution et pouvoir sont étudiés en considérant les nouveaux acteurs et technologies de la production cartographique. Pour les intercommunalités métropolitaines 
(Courmont) et les régions (Le Berre), la carte donne consistance aux territoires et, par la même, participe à la consolidation institutionnelle de ces nouveaux échelons et à leur projection dans le futur. Au niveau supranational, les programmes cartographiques financés par l'Union européenne donnent par exemple à voir tant les tensions sur le contenu et les modes de représentation de l'espace communautaire, que la constitution d'un savoir cartographique standardisé et autonome participant de la construction de l'Union (Zanin). Par ailleurs, ce pouvoir instituant des cartes passe par des technologies nouvelles de production et de représentation des données territoriales. Ainsi, si la mise en place d'un système d'information géographique (SIG) adossé à de nouvelles données informatisées est au tournant des années 1990 un mode d'affirmation de l'intercommunalité lyonnaise (Courmont), les données territoriales sont aujourd'hui de moins en moins l'apanage des pouvoirs publics qui ont perdu le monopole de l'expertise et de l'exploitation des données à l'époque du Big Data et de l'Open Data : des grandes entreprises voire des citoyens organisés collectivement prennent une place parfois importante dans leur production et leur mise en circulation (Mericskay).

La deuxième partie du livre s'intéresse aux modifications des cartes dans et pour le changement des politiques publiques. C'est dans les usages sociaux et politiques des cartes qu'il est possible d'en apprécier le rôle dans la définition et la mise à l'agenda des problèmes publics. Ainsi, l'évolution de l'objectivation cartographique d'une même question, telle que celle de l'approvisionnement alimentaire des villes états-uniennes, appuie et participe de la redéfinition du problème public et de la manière de le traiter (Larcher). Par ailleurs, comme le montrent les cas de la Politique agricole commune (Mesnel) et de la Politique de la Ville (Nonjon, O’Miel et Talpin), la modification des découpages et des zonages territoriaux est souvent justifiée par les réformateurs au nom de la «rationalisation » de l'action publique. Ces enquêtes multiniveaux établissent que l'imposition de nouvelles cartographies transforme en profondeur les modes d'allocation et de distribution des ressources publiques au niveau local. Ici, les modalités concrètes prises par ces cartes participent tantôt à en faire des «instruments automatiques » adossés à des calculs statistiques et faiblement politisés, tantôt à les construire en objet de contestation. Au prisme des usages dont elle fait l'objet dans le changement d'action publique, la carte est alors non seulement un outil de gouvernement, mais également une ressource politique pour des acteurs proposant des politiques alternatives, comme dans le cas des revendications bretonnes étudiées par J. Toutous autour du redécoupage des régions.

La troisième partie du livre déplace ensuite la focale sur les acteurs intermédiaires et de terrain, participant à la mise en œuvre de l'action publique au moment où les zonages et les outils cartographiques sont refaçonnés ou créés de toute pièce. Ces analyses «par le bas » montrent que les acteurs sont en fait confrontés à une pluralité de cartes parfois en contradiction, relevant de 
secteurs, niveaux de gouvernement, ou divers et auxquels sont attachés des d'intérêts spécifiques (groupes sociaux, bureaucraties, etc.). Une analyse fine de domaines d'action publique aussi divers que la santé (Aranda et Froger-Lefebvre), la politique de la ville (Arnoulet) ou encore la gestion de l'eau (de la Croix et Verhaeghe) montre que ces cartes font l'objet d'ajustements et de résistances pour les adapter aux contextes locaux et les rendre ainsi maniables, opératoires et in fine en partie contrôlables par des acteurs pris dans des systèmes de contraintes et interdépendances fortes.

La quatrième et dernière partie de l'ouvrage s'intéresse enfin aux zonages des politiques publiques, notamment dans les domaines éducatif et social, en lien avec les inégalités socioéconomiques et spatiales. D'une part, la question des inégalités ne peut pas être séparée de celle de l'accès aux services et aux droits. En effet, les choix de localisation participent à structurer les conditions de possibilité d'accès par des publics bénéficiaires, eux-mêmes dotés de ressources individuelles inégales (Deville), et la difficulté d'accès participe avec d'autres facteurs de la mise en échec des politiques publiques, comme dans le cas du décrochage scolaire (Caro, Checcaglini, Lepetit). D'autre part, ces chapitres montrent la nécessité de déconstruire les critères sous-jacents aux cartes, tels que le «modèle de l'accessibilité » pour l'accès aux droits sociaux (qui repose sur un postulat de mobilité et autonomie des bénéficiaires) (Deville) ou le choix de paramètres territoriaux (et non ethno-raciaux) dans les zonages de la géographie prioritaire (Epstein). Comme l'éclaire entre autres ce dernier cas, ces découpages font l'objet de représentations, de discours et d'attentes essentiels pour comprendre la légitimation de l'action publique, les critiques dont elle fait l'objet mais aussi ses conséquences éventuelles sur les publics. 